genes. In addition, treatment with pro-inflammatory cytokines and LPS caused ER stress and induced cleavage, and therefore activation, of CREBH in the liver of wild-type mice.

Finally, because CREBH and ATF6 are cleaved by the same proteases and because both have basic leucine-zipper domains, the authors proposed that they might form dimers. Indeed, immunoprecipitation studies of hepatocytes treated with an ER-stress inducer revealed the formation of heterodimers of the cleaved forms of CREBH and ATF6, and this resulted in synergistic activation of target-gene expression.

These results might be relevant to the pathogenesis of atherosclerosis, which is associated with ER stress and increased levels of serum CRP.

ORIGINAL RESEARCH PAPER Zhang, K. et al. Endoplasmic reticulum stress activates cleavage of CREBH to induce a systemic inflammatory response. Cell 124, 587-599 (2006)

same inhibitor could also completely abrogate the increased IL-10 production, indicating an important role for MKP1 in providing a negative signal for $\mathrm{p} 38$ activation in response to LPS. In addition, LPS-induced phosphorylation of the MAPK JNK (JUN amino-terminal kinase) was also increased and sustained in $\mathrm{Mkp1}^{-1-}$ cells, which indicates a negative role for MKP1 in JNK phosphorylation.

These studies show that MKP1 has an important role in controlling MAPK-regulated inflammation in vivo and functions as a key regulator of both pro- and anti-inflammatory cytokines in response to TLR signals.

Olive Leavy

ORIGINAL RESEARCH PAPERS Chi, H. et al. Dynamic regulation of pro- and anti-inflammatory cytokines by MAPK phosphatase 1 (MKP-1) in innate immune responses. Proc. Natl Acad. Sci. USA 103. 2274-2279 (2006) | Hammer, M. et al. Dual specificity phosphatase 1 (DUSP1) regulates a subset of LPS-induced genes and protects mice from lethal endotoxin shock. J. Exp. Med. 203, 15-20 (2006) | Salojin, K. V. et al. Essential role of MAPK phosphatase-1 in the negative control of innate immune responses. J. Immunol. 176, 1899-1907 (2006)| Zhao, Q. et al. MAP kinase phosphatase 1 controls innate immune responses and suppresses endotoxic shock. J. Exp. Med. 203, 131-140 (2006)

\title{
Recognition of cytosolic DNA
}

Recognition of viral nucleic acids by the endosomal Toll-like receptors (that is, TLR3, TLR7, TLR8 and TLR9), and by the cytosolic RNA helicases RIG-I (retinoic-acid-inducible gene I) and MDA5 (melanoma differentiation-associated protein 5) triggers the production of type I interferons (IFN $\alpha$ and IFN $\beta$ ). The NOD (nucleotide-binding oligomerization domain) proteins are another type of cytosolic sensor of microbial components. Now, in a study published in Immunity, Daniel Stetson and Ruslan Medzhitov describe a new pathway by which the recognition of cytosolic DNA leads to the production of type I IFNs.

Some evidence already existed to indicate that the production of type I IFNs could be induced by infection with Listeria monocytogenes - a Gram-positive bacterium that grows in the cytosol - in a TLR- and NOD-independent manner, but the triggering ligand was unknown. So, the authors set out to determine whether there is a pathway for cytosolic recognition of DNA, equivalent to the recognition of RNA by RIG-I and MDA5. They used cells from two types of double-knockout mice: macrophages from mice that lack MyD88 (myeloid differentiation primary-response gene 88 ) and TRIF (Toll/ interleukin-1 (IL-1)-receptor-domain-containing adaptor protein inducing IFN $\beta$ ), which cannot transmit signals through any of the TLRs, and macrophages from mice that lack MyD88 and RIP2 (receptor-interacting protein 2), which lack both TLR and NOD-protein signalling. In response to infection with $L$. monocytogenes, the induction of IFN $\beta$ and IL- 6 production by macrophages from both types of mice was intact. However, L. monocytogenes extracts that were pre-treated with DNase had an impaired ability to induce IFN $\beta$ and IL- 6 production, whereas extracts that were treated with RNase behaved as untreated extracts, indicating a role for recognition of bacterial DNA.

Next, the authors showed that a pathogen that is confined to a host-cell vacuole could also trigger this pathway. Once phagocytosed by the host cell, Legionella pneumophila establishes a replicative organelle by injecting proteins into the host-cell cytosol, through a Dot/Icm type IV secretion system, that block phagosome-

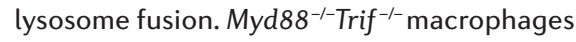
infected with opsonized L. pneumophila produced both IFN $\beta$ and IL- 6 but were unable to do so when infected with mutant bacteria that lacked the type IV secretion system. As this secretion system can transfer DNA

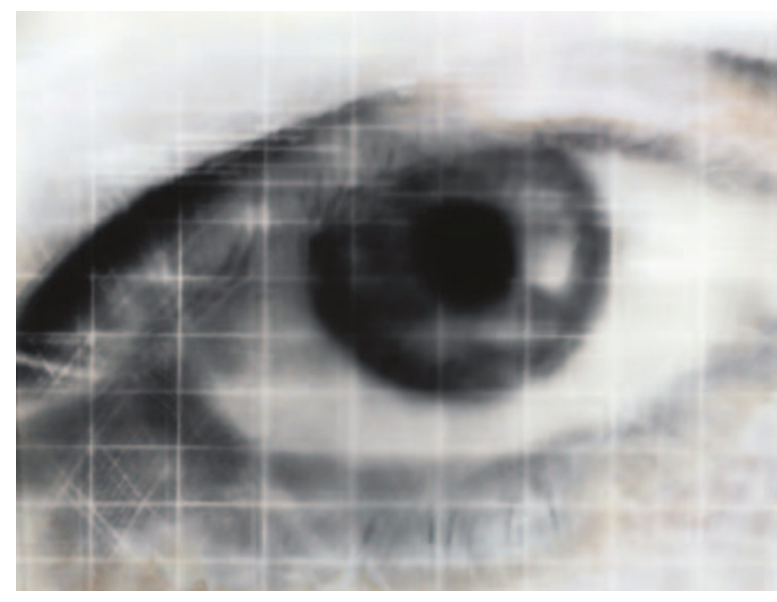

as well as proteins, it is possible that transferred DNA is recognized in the cytosol and induces cytokine production.

As TLR9 recognizes $\mathrm{CpG}$ motifs present in microbial DNA, the authors used DNA that lacks CpG motifs (referred to as interferon stimulatory DNA, ISD) to look at DNA-induced responses independently of TLR9 recognition. ISD induced type I IFN production in TLR9-deficient cells in a sequence-independent manner. An intact sugarphosphate backbone was found to be essential for recognition of ISD. Consistent with recognition of ISD in the cytosol, the authors found that chloroquine treatment (which inhibits endosomal acidification and prevents TLR9 activation) of macrophages abrogated CpG DNA-induced type I IFN production but had no effect on type I IFN production stimulated by ISD.

Further experiments showed that ISD induces type I IFN production independently of signalling through the nuclear factor- $\mathrm{KB}$ or mitogen-activated protein kinase pathways. Finally, gene-expression analysis using microarrays showed that recognition of DNA in the cytosol (ISD) and endosomes (CpG DNA) activated distinct but overlapping gene-expression programmes.

Taken together, these results provide evidence of a novel pathway for recognition of cytosolic DNA. The next step will be to identify the DNA sensor and further compare the innate immune response pathways for DNA recognition.

\section{Elaine Bell}

ORIGINAL RESEARCH PAPER Stetson, D. B. \& Medzhitov, R. Recognition of cytosolic DNA activates an IRF3-dependent innate immune response. Immunity 24, 93-103 (2006) 